\title{
Assessment of Prenatal Kynurenine Metabolism Using Tissue Slices: Focus on the Neosynthesis of Kynurenic Acid in Mice
}

\author{
Francesca M. Notarangelo Sarah Beggiato Robert Schwarcz \\ Maryland Psychiatric Research Center, Department of Psychiatry, University of Maryland School of Medicine, Baltimore, \\ MD, USA
}

\section{Keywords \\ Development $\cdot$ Kynurenine $\cdot$ Schizophrenia}

\begin{abstract}
Several lines of evidence support the hypothesis that abnormally elevated brain levels of kynurenic acid (KYNA), a metabolite of the kynurenine pathway (KP) of tryptophan degradation, play a pathophysiologically significant role in schizophrenia and other major neurodevelopmental disorders. Studies in experimental animal models suggest that KP impairments in these diseases may originate already in utero since prenatal administration of KYNA's bioprecursor, kynurenine, leads to biochemical and structural abnormalities as well as distinct cognitive impairments in adulthood. As KP metabolism during pregnancy is still insufficiently understood, we designed this study to examine the de novo synthesis of KYNA and 3-hydroxykynurenine (3-HK), an alternative biologically active product of kynurenine degradation, in tissue slices obtained from pregnant mice on gestational day (GD) 18. Fetal brain and liver, placenta, and maternal brain and liver were collected, and the tissues were incubated in vitro in the absence or presence of micromolar concentrations of kynurenine. KYNA and 3-HK were measured in the
\end{abstract}

extracellular milieu. Basal and newly produced KYNA was detected in all cases. As KYNA formation exceeded 3-HK production by $2-3$ orders of magnitude in the placenta and maternal brain, and as very little 3-HK neosynthesis was detectable in fetal brain tissue, detailed follow-up experiments focused on KYNA only. The fetal brain produced 3-4 times more KYNA than the maternal brain and placenta, though less than the maternal and fetal liver. No significant differences were observed when using tissues obtained on GD 14 and GD 18. Pharmacological inhibition of KYNA's main biosynthetic enzymes, kynurenine aminotransferase (KAT) I and KAT II, revealed qualitative and quantitative differences between the tissues, with a preferential role of KATI in the fetal and maternal brain and of KAT II in the fetal and maternal liver. Findings using tissue slices from KAT II knockout mice confirmed these conclusions. Together, these results clarify the dynamics of KP metabolism during pregnancy and provide the basis for the conceptualization of interventions aimed at manipulating cerebral KP function in the prenatal period.

(c) 2019 S. Karger AG, Basel

Francesca M. Notarangelo and Sarah Beggiato contributed equally to the study.

\section{KARGER}

() 2019 S. Karger AG, Basel

E-Mail karger@karger.com

www.karger.com/dne
Francesca M. Notarangelo, $\mathrm{PhD}$

Maryland Psychiatric Research Center

PO Box 21247

Baltimore, MD 21228 (USA)

E-Mail fnotarangelo@ som.umaryland.edu 


\section{Introduction}

The kynurenine pathway (KP) is the main catabolic route of the essential amino acid tryptophan in mammals. Kynurenine, the first stable KP metabolite (Fig. 1), generates several neuroactive metabolites downstream $[1,2]$. One of these compounds, kynurenic acid (KYNA), which is produced primarily by irreversible enzymatic transamination of kynurenine $[3,4]$, is an endogenous inhibitor of $\alpha 7$ nicotinic acetylcholine ( $\alpha 7 \mathrm{nACh}$ ) and $N$-methyl-Daspartate (NMDA) receptors, and also affects other specific targets $[5,6]$. In humans, rats, and mice, four kynurenine aminotransferases (KAT I-IV) are able to catalyze the formation of KYNA, with KAT I and especially KAT II considered to be most relevant for rapid synthesis in the brain and liver of adult rats and humans $[4,7]$. Notably, no mechanisms for KYNA degradation appear to exist in mammals [1], and newly synthesized KYNA is promptly liberated into the extracellular milieu [8-10]. In a separate branch of the KP, kynurenine is first degraded to the free radical generator, 3-hydroxykynurenine (3$\mathrm{HK})$, by kynurenine 3-monooxygenase (KMO), and then further to a series of biologically active metabolites, eventually leading to the formation of the abundant cofactor $\mathrm{NAD}^{+}[1,2]$. Kynurenine therefore occupies a pivotal position in the KP, making the ratio between newly produced KYNA and 3-HK an informative marker of the function of the entire metabolic cascade.

Malfunction of the KP has been plausibly linked to the pathophysiology of several diseases, including a number of psychiatric and neurological disorders $[1,2,11-15]$. Studied most extensively in this regard, elevated KYNA levels (but not 3-HK levels) have been found in the postmortem brain and cerebrospinal fluid of individuals with schizophrenia, a major brain disease that originates early in life [16-20]. In experimental animals, increased brain KYNA concentrations cause several cognitive impairments, which are remarkably similar to those seen in people with schizophrenia [1, 21-25]. Notably, and in line with the neurodevelopmental etiology of the disease, prolonged administration of kynurenine to pregnant rats results in elevated brain KYNA (but not 3-HK) levels, as well as distinct cognitive abnormalities, in adult offspring [26] .

Interestingly, the mammalian brain normally contains several-fold higher concentrations of kynurenine, KYNA, and 3 -HK during the fetal period than at any postnatal stage [27-31]. As the cerebral levels of all three of these KP metabolites decrease precipitously immediately after birth $[28,31]$, it is tempting to surmise that they normally serve distinct roles in prenatal brain development. This

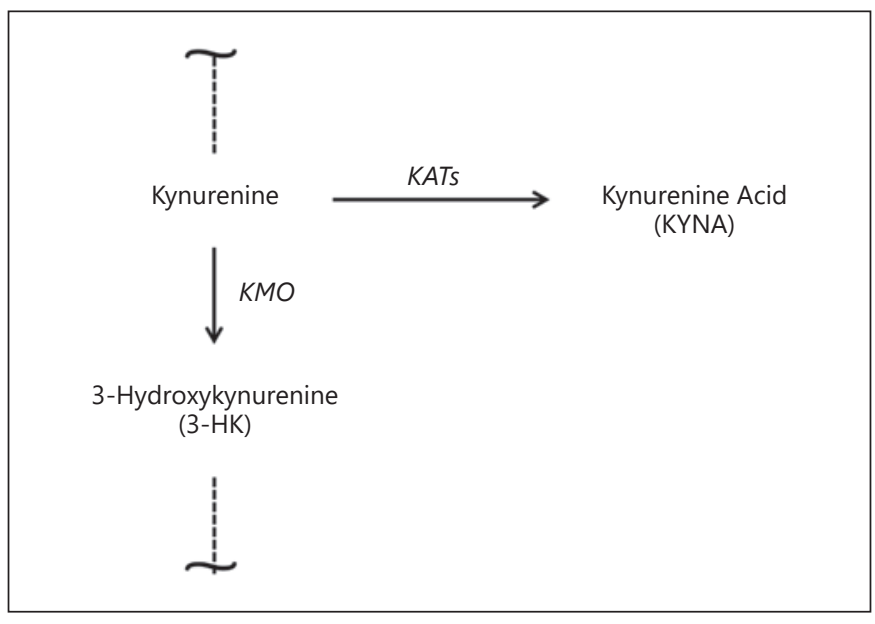

Fig. 1. Simplified illustration of the branching point of the kynurenine pathway of tryptophan degradation. KATs, kynurenine aminotransferases; KMO, kynurenine 3-monooxygenase.

assumption is indirectly supported by evidence that an impairment of KP metabolism during the prenatal period has adverse consequences [26,32-38], but has not been sufficiently evaluated so far.

In contrast to maternally derived kynurenine and 3-HK, maternal KYNA is not able to cross the placenta [39]. As the administration of tryptophan or kynurenine to pregnant dams readily raises KYNA levels in the fetal brain $[26,32,39,40]$, and as the placenta plays only a minor role in this process [39], fetal KYNA must be synthesized locally. The present study was designed to provide further insights by elaborating the characteristics of the prenatal conversion of kynurenine to KYNA and 3-HK, respectively, ex vivo. To this end, we collected the maternal brain and liver, placenta, and fetal brain and liver from pregnant mice on gestational day (GD) 18, and in some cases on GD 14. Slices from the various tissues were then incubated in vitro in the absence or presence of kynurenine, and basal levels and neosynthesis of KYNA and 3 -HK were assessed in the extracellular milieu. By elucidating the features of two critical KP branches during pregnancy, our experimental results provided valuable new insights into the dynamics and, by implication, the function of prenatal KP metabolism.

\section{Materials and Methods}

\section{Chemicals}

KYNA, ascorbic acid, aminooxyacetic acid (AOAA), and glutamine were purchased from Sigma-Aldrich (St. Louis, MO, USA). L-kynurenine sulfate ("kynurenine"; purity: 99.4\%) was 
obtained from Sai Advantium (Hyderabad, India). The selective KAT II inhibitor BFF-122 [(S)-(-)-9-(4-aminopiperazine-1-yl)8-fluoro-3-methyl-6-oxo-2, 3, 5, 6-tetrahydro-4H-1-oxa-3a-azaphenalene-5-carboxylic acid] was kindly provided by Dr. Y. Kajii (Mitsubishi-Tanabe Pharma Corp., Yokohama, Japan). The KMO inhibitor Ro 61-8048 was a generous gift from Dr. W. Fröstl (Novartis, Basel, Switzerland). All other chemicals were obtained from various commercial suppliers and were of the highest available purity.

\section{Animals}

Wild-type (WT) FVB/N mice (8-9 weeks) were purchased from Jackson Laboratories (Bar Harbor, ME, USA). KAT II knockout (KO) FVB/N mice were generated as previously described [41]. Male and female mice were mated in-house for up to $48 \mathrm{~h}$, and the presence of a copulation plug was confirmed on GD 1 or GD 2. The male was then removed, and the female was left undisturbed until the day of the experiment.

Animals were maintained on a $12 \mathrm{~h} / 12 \mathrm{~h}$ light/dark cycle in a temperature-controlled room in the animal facility of the Maryland Psychiatric Research Center. Access to food and water was provided ad libitum.

\section{Tissue Preparation and Incubation}

On the day of the experiment, pregnant mice $(n=3-6$ per group) were euthanized with $\mathrm{CO}_{2}$, and the maternal and fetal brains and livers as well as the placenta were rapidly removed and placed in oxygenated Krebs-Ringer buffer (117.6 mM NaCl, 4.6 $\mathrm{mM} \mathrm{KCl}, 2.4 \mathrm{mM} \mathrm{CaCl}_{2}, 2.4 \mathrm{mM} \mathrm{MgSO}_{4}, 3.1 \mathrm{mM} \mathrm{NaH}_{2} \mathrm{PO}_{4}, 26.2$ $\mathrm{mM} \mathrm{NaHCO}, 11.1 \mathrm{~mm}$ glucose, $\mathrm{pH} 7.4)$. Ascorbic acid $(500 \mu \mathrm{M})$ was added to the buffer to avoid non-enzymatic oxidation of kynurenine and its metabolites.

Tissue slices ( $0.4 \mathrm{~mm}$ thick) were prepared from the maternal forebrain, the entire placenta, the whole fetal brain, one lobe of the maternal liver and the whole fetal liver, using a McIIwain chopper (Mickle Laboratory Engineering, Gomshall, UK). The tissues were immediately immersed in Krebs-Ringer buffer for $20 \mathrm{~min}$, and one slice each from maternal brain, liver, or placenta was placed into a well containing $200 \mu \mathrm{L}$ of buffer, using a multiwell culture plate. Fetal brain and liver slices were directly taken from the oxygenated buffer with a pipette and transferred into the wells (final volume: $200 \mu \mathrm{L}$ ) [42]. Twenty $\mu \mathrm{L}$ of buffer or of a solution containing kynurenine (final concentration: 10 or $100 \mu \mathrm{M}$ ) were added, and the tissues were incubated for $1 \mathrm{~h}$ at $37^{\circ} \mathrm{C}$ in a shaking water bath. Separate wells were incubated with kynurenine in the absence of tissue. The culture plate was then placed on ice, and the reaction was terminated by adding $25 \mu \mathrm{L}$ of $2 \mathrm{~N} \mathrm{HCl}$ and $25 \mu \mathrm{L}$ of $25 \%$ perchloric acid to each well. The medium was rapidly removed and centrifuged $(6,000 \mathrm{~g}, 5 \mathrm{~min})$, and the supernatant was subjected to HPLC analysis for KYNA or 3-HK measurement (see below). Tissue slices were homogenized in $200 \mu \mathrm{L}$ of ultrapure water and frozen at $-80^{\circ} \mathrm{C}$ for protein determination. All assays were performed in duplicate or triplicate. De novo KYNA or 3-HK production was determined by subtracting both endogenous levels (incubation of tissue in the absence of kynurenine) and levels measured following incubation of kynurenine alone (no tissue).

The effect of KAT inhibition was determined using the nonspecific aminotransferase inhibitor AOAA (1 mM), the KAT I inhibitor glutamine (1 mM), or the specific KAT II inhibitor BFF-122 (1 $\mathrm{mM})$ (all final concentrations). These compounds were added
$(20 \mu \mathrm{L})$ to the incubation mixture together with kynurenine (10 $\mu \mathrm{M})$. Controls were obtained by incubating the compounds without tissue.

\section{KYNA Analysis}

For KYNA determination, $20 \mu \mathrm{L}$ of the supernatant were applied to a $3-\mu \mathrm{m}$ C18 reverse-phase HPLC column $(100 \times 4 \mathrm{~mm}$; Dr. Maisch $\mathrm{GmbH}$, Ammerbuch, Germany), using a mobile phase containing $50 \mathrm{~mm}$ sodium acetate and $3 \%$ acetonitrile (pH adjusted to 6.2 with glacial acetic acid) at a flow rate of $0.5 \mathrm{~mL} / \mathrm{min}$. Zinc acetate $(0.5 \mathrm{M}$, not $\mathrm{pH}$-adjusted) was delivered post-column by a peristaltic pump (Dionex AXP, Thermo Fisher, Waltham, MA, USA) at a flow rate of $0.1 \mathrm{~mL} / \mathrm{min}$. In the eluate, KYNA was detected fluorimetrically (excitation: $344 \mathrm{~nm}$, emission: $398 \mathrm{~nm}$; S200a fluorescence detector; Perkin Elmer, Waltham, MA, USA). The retention time of KYNA was approximately $14 \mathrm{~min}$.

\section{3-HK Analysis}

For 3-HK determination, $20 \mu \mathrm{L}$ of the supernatant were applied to a $3-\mu \mathrm{m}$ C18 reverse-phase HPLC column (HR-80; $80 \times 4.6 \mathrm{~mm}$; Thermo Fisher). The mobile phase consisted of $1.5 \%$ acetonitrile, $0.9 \%$ trimethylamine, $0.59 \%$ phosphoric acid, $0.27 \mathrm{~mm}$ EDTA, and $8.9 \mathrm{~mm}$ sodium heptane sulfonic acid. 3 -HK was eluted at a flow rate of $0.5 \mathrm{~mL} / \mathrm{min}$ and detected electrochemically using an HTEC 500 detector (Eicom, San Diego, CA, USA; oxidation potential: + $0.5 \mathrm{~V})$. The retention time of $3-\mathrm{HK}$ was approximately $11 \mathrm{~min}$.

\section{KMO Enzyme Activity}

To determine KMO activity, the fetal $(1: 5, \mathrm{w} / \mathrm{v})$ and maternal $(1: 25, \mathrm{w} / \mathrm{v})$ brains were homogenized in $100 \mathrm{~mm}$ Tris- $\mathrm{HCl}$ buffer ( $\mathrm{pH} 8.1$ ) containing $10 \mathrm{mM} \mathrm{KCl}$ and $1 \mathrm{mM}$ EDTA. Eighty $\mu \mathrm{L}$ of the preparation were then incubated for $40 \mathrm{~min}$ at $37^{\circ} \mathrm{C}$ in a solution containing $1 \mathrm{~mm}$ NADPH, $3 \mathrm{~mm}$ glucose-6-phosphate, $1 \mathrm{U} / \mathrm{mL}$ glucose- 6 phosphate dehydrogenase, $100 \mu \mathrm{M}$ L-kynurenine, $10 \mathrm{mM}$ $\mathrm{KCl}$, and $1 \mathrm{mM}$ EDTA in a total volume of $200 \mu \mathrm{L}$. The reaction was stopped by the addition of $50 \mu \mathrm{L}$ of $6 \%$ perchloric acid. Blanks were obtained by adding the KMO inhibitor Ro 61-8048 (100 $\mu \mathrm{M})$ to the incubation solution. After centrifugation $(16,000 \mathrm{~g}, 15 \mathrm{~min})$, $20 \mu \mathrm{L}$ of the resulting supernatant were subjected to HPLC analysis to measure $3-\mathrm{HK}$, as described above.

\section{Protein Measurement}

Protein was measured according to the method of Lowry et al. [43] using bovine serum albumin as a standard.

\section{Statistical Analysis}

All data are expressed as the mean \pm SEM. Student's $t$ test, oneway or two-way ANOVA, followed by Bonferroni's post hoc test were used to determine significance in all experiments. A $p$ value $<0.05$ was considered significant.

\section{Results}

\section{Basal Levels and de novo Production of 3-HK and} KYNA: Maternal Brain, Placenta, and Fetal Brain

We first compared the status of $3-\mathrm{HK}$ and KYNA in the maternal brain, placenta, and fetal brain of WT mice at 
Table 1. Basal level and de novo production of extracellular 3-HK and KYNA in maternal brain, placenta, and fetal brain

\begin{tabular}{|c|c|c|c|c|}
\hline & & \multirow[t]{2}{*}{ Basal level } & \multicolumn{2}{|l|}{ De novo production } \\
\hline & & & $10 \mu \mathrm{M}$ kynurenine & $100 \mu \mathrm{M}$ kynurenine \\
\hline \multirow{2}{*}{ Maternal brain } & 3-HK (pmol/h/mg protein) & $0.21 \pm 0.03$ & 0 & $0.18 \pm 0.12$ \\
\hline & KYNA (pmol/h/mg protein) & $0.13 \pm 0.06$ & $2.67 \pm 0.56$ & $22.50 \pm 4.58$ \\
\hline \multirow[t]{2}{*}{ Placenta } & 3-HK (pmol/h/mg protein) & $0.33 \pm 0.11$ & 0 & $0.63 \pm 0.20$ \\
\hline & KYNA (pmol/h/mg protein) & $1.12 \pm 0.23$ & $1.23 \pm 0.44$ & $20.44 \pm 4.06$ \\
\hline \multirow[t]{2}{*}{ Fetal brain } & 3-HK (pmol/h/mg protein) & $1.97 \pm 0.27$ & 0 & $0.04 \pm 0.29$ \\
\hline & KYNA (pmol/h/mg protein) & $0.93 \pm 0.09$ & $7.34 \pm 1.23$ & $88.78 \pm 14.42$ \\
\hline
\end{tabular}

Basal extracellular level and de novo production of 3-HK and KYNA in tissue slices obtained from GD 18 WT mice. Neosynthesis was assessed by incubation in the presence of 10 or $100 \mu \mathrm{M}$ kynurenine and was calculated by subtracting basal level. Data are the mean \pm SEM $(n=2-6)$. See the text for experimental details and statistical analyses.

GD 18 under physiological conditions. Both metabolites were detected in the media of all 3 tissues following the $1-\mathrm{h}$ incubation (Table 1). Under these basal conditions, 3-HK levels were several-fold higher in the medium obtained from the fetal brain than from either the maternal brain or the placenta (each $p<0.001$, two-way ANOVA followed by Bonferroni's post hoc test). Measured in the same samples as 3-HK, extracellular KYNA concentrations recovered from placental and fetal brain slices were much higher than those recovered from maternal brain tissue ( $p<0.001$ and $p<0.01$, respectively; two-way ANOVA followed by Bonferroni's post hoc test). Notably, under these basal conditions, fetal brain slices released more 3 -HK than KYNA ( $p<0.001$, two-way ANOVA followed by Bonferroni's post-hoc test), whereas the placenta released more KYNA than 3-HK ( $p<0.05$, two-way ANOVA followed by Bonferroni's post hoc test). Medium obtained after the incubation of maternal brain slices showed no preference for either metabolite (Table 1).

In all three tissues, de novo production of KYNA from kynurenine exceeded the neosynthesis of 3-HK (Table 1). Specifically, incubation with 10 and $100 \mu \mathrm{M}$ of the common bioprecursor kynurenine increased KYNA levels linearly, whereas 3-HK formation was very limited. In fact, only small increases in extracellular 3-HK levels were seen, even after the tissues were exposed to $100 \mu \mathrm{M}$ of kynurenine. Notably, incubation with kynurenine caused fetal brain slices to release several times more KYNA than the two other tissues (each $p<0.001$, two-way ANOVA followed by Bonferroni's post hoc test). There were no clear differences in the minor de novo production of 3-HK between the tissues tested.

Prenatal KP Metabolism Using Tissue Slices: Neosynthesis of KYNA in Mice
Separately, we determined the activity of KMO, which catalyzes 3-HK formation from kynurenine (Fig. 1), in tissue homogenates. This experiment revealed a significantly lower enzyme activity in the fetal brain $(0.20 \pm 0.05$ $\mathrm{pmol} 3-\mathrm{HK} / \mathrm{h} / \mathrm{mg}$ tissue; $n=3)$ than in the maternal brain $(2.59 \pm 0.53 \mathrm{pmol} 3-\mathrm{HK} / \mathrm{h} / \mathrm{mg}$ tissue; $n=3 ; p<0.01$, Student's $t$ test), supporting the conclusion that the fetal brain has only a limited capacity to produce 3 -HK from its direct bioprecursor.

In view of these findings, subsequent experiments focused exclusively on the neosynthesis of KYNA. To approximate physiological conditions in fetal tissues [28, 44], $10 \mu \mathrm{M}$ of kynurenine was routinely used to drive KYNA production in all following studies.

\section{Basal Levels and de novo Production of KYNA: Fetal and Maternal Liver}

Next, we determined extracellular KYNA following the incubation of tissue slices from fetal and maternal liver (Table 2). Less KYNA was detected in the media obtained from fetal than from maternal tissue $(p<0.01$, Student's $t$ test). Still, the basal extracellular KYNA levels recovered from the fetal liver were substantially higher than those measured after the incubation of placental or fetal brain slices (each $p<0.001$, one-way ANOVA followed by Bonferroni's post hoc test) (Table 1).

Incubation of fetal and maternal liver slices with $10 \mu \mathrm{M}$ of kynurenine significantly raised extracellular KYNA levels in both cases (each $p<0.01$ vs. basal levels, Student's $t$ test), and the effect was greater in the maternal tissue ( $p<0.05$, Student's $t$ test). However, as observed for basal values, even KYNA neosynthesis in the fetal liver great-

Dev Neurosci 2019;41:102-111 


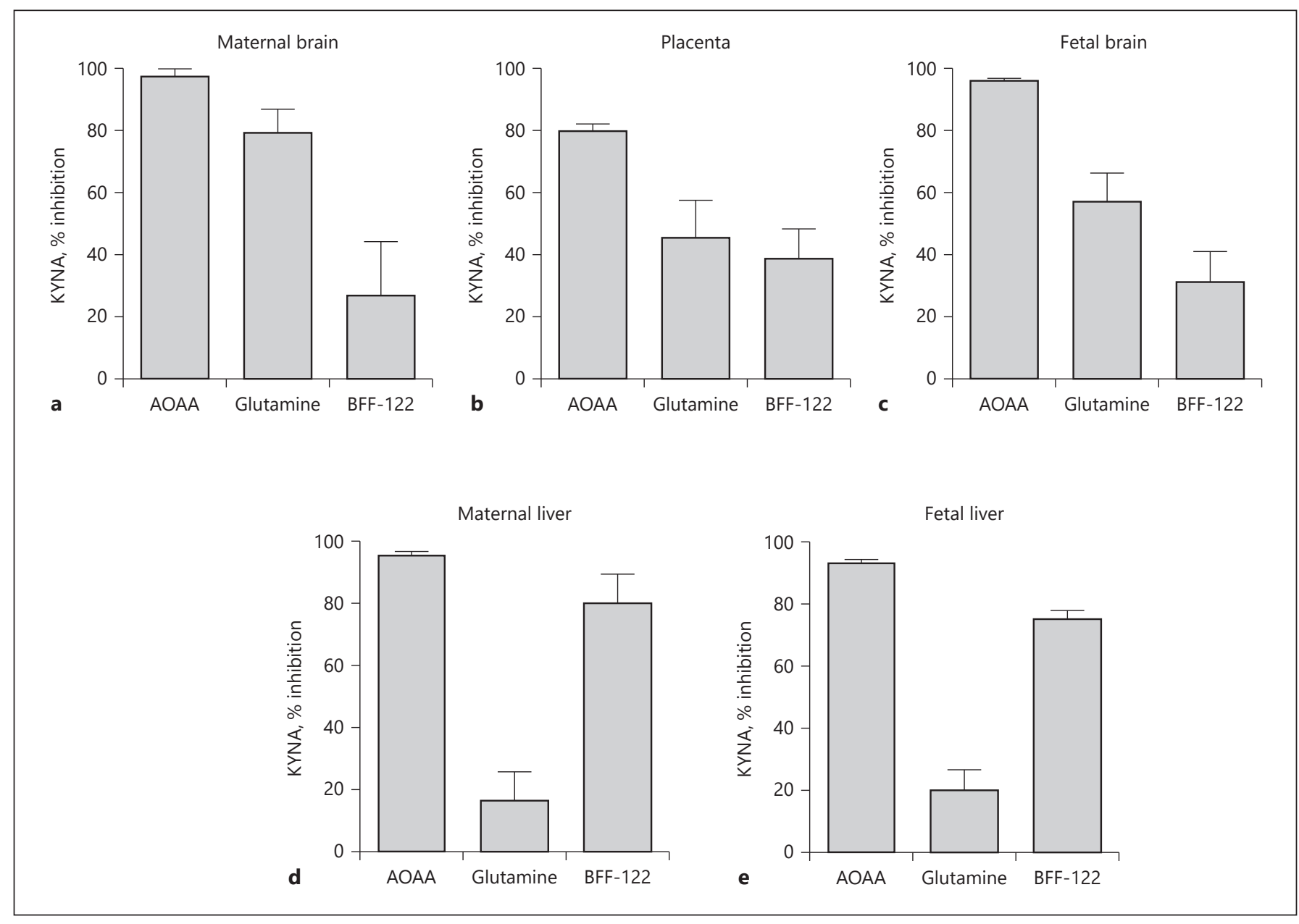

Fig. 2. Effects of KAT inhibition on the neosynthesis of KYNA in slices from maternal brain (a), placenta (b), fetal brain (c), maternal liver (d), and fetal liver (e), obtained from wild-type (WT) mice on gestational day (GD) 18 (absolute values, Tables 1, 2). Tissues were incubated in the presence of $10 \mu \mathrm{M}$ kynurenine for $1 \mathrm{~h}$ at

Table 2. Basal level and de novo production of extracellular KYNA in fetal and maternal liver

\begin{tabular}{lcc}
\hline & Fetal liver & $\begin{array}{l}\text { Maternal } \\
\text { liver }\end{array}$ \\
\hline Basal level, pmol/h/mg protein & $3.5 \pm 0.4$ & $9.7 \pm 1.1$ \\
De novo production, pmol/h/mg protein & $40.0 \pm 7.3$ & $91.3 \pm 19.1$
\end{tabular}

Basal extracellular level and de novo production of KYNA after incubation of tissue slices from fetal and maternal liver of GD 18 WT mice with $10 \mu \mathrm{M}$ kynurenine. De novo production indicates values obtained after the subtraction of basal level. Data are the mean \pm SEM $(n=4)$. See the text for experimental details and statistical analyses. $37^{\circ} \mathrm{C}$, and experiments were performed as described in the text. AOAA, general KAT inhibitor; glutamine, KAT I inhibitor; BFF122, selective KAT II inhibitor. Final concentration of all three agents: $1 \mathrm{mM}$. Data are the mean \pm SEM $(n=4-5)$.

ly exceeded de novo production in the placenta and fetal brain (each $p<0.001$, one-way ANOVA followed by Bonferroni's post hoc test).

\section{Pharmacological Inhibition of KYNA Synthesis}

To investigate the role of KAT enzymes in KYNA neosynthesis, we tested the effects of the non-specific aminotransferase inhibitor AOAA, glutamine (which inhibits KAT I), and the specific KAT II inhibitor BFF-122 [7, 45, 46].

The pharmacological tools used revealed qualitative differences between the tissues analyzed (Fig. 2). Thus, whereas incubation in the presence of AOAA almost completely prevented the de novo production of KYNA 




Fig. 3. Basal extracellular levels (a) and de novo KYNA production from kynurenine $(10 \mu \mathrm{M} ; \mathbf{b})$, determined in the extracellular milieu of tissue slices from maternal brain, placenta, fetal brain, maternal liver, and fetal liver of KAT II knockout (KO) mice (on GD 17/18) following incubation for $1 \mathrm{~h}$ at $37^{\circ} \mathrm{C}$. Data are expressed as $\%$ of the levels in WT mice (absolute values, Tables 1, 2). Experiments were performed as described in the text. De novo production was calculated by subtracting basal levels. Data are the mean \pm SEM $(n=3-5)$. See the text for statistical analyses. in all tissues tested, KYNA synthesis in the maternal and fetal brain was preferentially reduced by KAT I inhibition (75 and 57\%, respectively; Fig. 2a, c). In contrast, KYNA formation in the maternal and fetal liver was mainly KAT II-dependent, as indicated by 80 and $75 \%$ inhibition, respectively, in the presence of BFF-122 (Fig. 2d, e). The placenta showed a mixed profile, with glutamine causing $45 \%$ inhibition of KYNA synthesis and BFF-122 causing 39\% inhibition (Fig. 2b).

\section{Studies Using Tissues from KAT II KO Mice}

Using standard experimental conditions, we next examined KYNA formation in the absence or presence of $10 \mu \mathrm{M}$ of kynurenine in tissue slices prepared from KAT II KO mice.

Following incubation of $\mathrm{KO}$ tissues under basal conditions, KYNA was always readily detectable in the extracellular milieu, albeit mostly at lower levels than in the corresponding samples from the WT mice (Fig. 3a). Specifically, slices prepared from KO mice produced 2 -fold (placenta and fetal brain) to 4 -fold (fetal and maternal liver) less KYNA than the corresponding tissues from WT animals (Tables 1, 2). However, there was no difference between the amount of KYNA released from maternal brain slices from WT and KAT II KO mice.

Incubation with kynurenine increased KYNA synthesis in fetal and maternal brains (each $p<0.05$, Student's $t$ test) and livers ( $p<0.001$. Student's $t$ test) of KAT II KO mice, but had only a small, non-significant effect in the placenta. Overall, de novo KYNA production in all tissues was lower than in the corresponding tissues from WT mice (Fig. 3b; Tables 1, 2), with the greatest reduction seen in fetal and maternal liver. The genotype difference reached statistical significance in the fetal $(p<0.01$, Student's $t$ test) and maternal ( $p<0.05$, Student's $t$ test) liver as well as in the fetal brain ( $p<0.05$, Student's $t$ test).

Taken together with the pharmacological data, these results demonstrate that, in mice, KAT II is of relatively little relevance in the maternal brain and only partially contributes to KYNA formation in the placenta and fetal brain but plays a major role in KYNA synthesis in the fetal and maternal liver.

\section{Basal Levels and de novo Production of KYNA at GD 14}

Finally, beginning to consider possible age-related differences during prenatal development, we conducted a pilot study examining KYNA production in WT mice at an earlier stage of gestation (GD 14). As in the preceding experiments, tissues were incubated for $1 \mathrm{~h}$, alone or in the presence of $10 \mu \mathrm{M}$ of kynurenine, and KYNA was measured in the extracellular milieu. Basal KYNA levels, expressed in $\mathrm{pmol} / \mathrm{mg}$ protein, were $0.06 \pm 0.03$ (maternal brain), $0.58 \pm 0.08$ (placenta), $0.83 \pm 0.15$ (fetal brain), $2.91 \pm 0.38$ (fetal liver) and $8.54 \pm 1.72$ (maternal liver) ( $n=3$ for each tissue). Statistical analyses did not reveal 
any differences between GD 14 and GD 18 in basal or newly formed KYNA in any of the tissues examined ( $p>$ 0.05 , Student's $t$ test).

\section{Discussion}

This study demonstrated that maternal, fetal, and placental tissues, all dissected from pregnant mice on GD 18, are capable of metabolizing kynurenine under physiological conditions in vitro. More specifically, our results revealed that the fetal brain, the major tissue of interest in our experiments, readily produces KYNA from kynurenine but is far less efficient in converting kynurenine to 3-HK. Most of our analyses therefore focused on KYNA formation. Using pharmacological and genetic approaches, we found significant differences between the processes that account for the de novo synthesis of KYNA in the various tissues. As kynurenine is the major catabolic product of the essential amino acid tryptophan, and as excessive prenatal exposure to kynurenine has adverse consequences later in life [26, 32-35, 37], we will discuss the implications of these results from both physiological and pathophysiological perspectives.

One of the major insights gained from the study was that the dynamics of KP metabolism on GD 18 differ both qualitatively and quantitatively, and in both the absence and the presence of kynurenine, between various tissues. Thus, slices from the fetal brain liberated substantially more 3 -HK than tissue from the placenta and the maternal brain into the extracellular milieu under basal conditions (defined here as a 1-h incubation in regular Krebs-Ringer buffer) but produced almost no 3-HK de novo when kynurenine was added to the incubation mixture. As the content of $3-\mathrm{HK}$ in the mammalian brain is several-fold higher prenatally than postnatally $[28,30]$, and as we detected only very little KMO activity in tissue homogenate prepared from the fetal brain, these findings indicate that the rapid accumulation of 3 -HK in the fetal brain seen following the administration of kynurenine to the dam in vivo $[39,47]$ is very likely not due to local synthesis but a consequence of increased influx of the metabolite from the dam. This interpretation is in line with the fact that the large neutral amino acid transporter LAT-1, which readily recognizes both kynurenine and 3-HK [48], is highly enriched in the placenta [49]. The placenta is therefore able to accumulate, and then transmit to the fetus, maternally derived 3-HK. Notably, however, and especially under inflammatory conditions that can activate KMO [1], 3-HK may also enter the fetus after it is formed in the placenta itself [50-54].

Parallel analyses of KYNA produced very different results. Under basal conditions, similar amounts of extracellular KYNA were recovered after incubating slices from the fetal brain and placenta, in both cases several times more than from maternal brain slices. However, placental slices were substantially less effective than fetal brain slices in producing KYNA from kynurenine de novo. Previous ex vivo work in mice [39], as well as studies using human placental extract [52], demonstrated that perfusion with kynurenine and tryptophan, respectively, has only a limited effect on KYNA formation within the placenta. Moreover, in vivo experiments revealed that approximately $40 \%$ of KYNA that appeared to derive from the placenta in fact originated from residual maternal blood [39].

Taken together, these results indicate that KYNA synthesis within the placenta is not likely to play a defining role in regulating KYNA levels in the fetus. Notably, acute KYNA neosynthesis in the fetal brain also exceeded KYNA formation and release from maternal brain tissue slices 3- to 5-fold. Although the fetal liver, which was found here to have an even greater ability to form KYNA from kynurenine, may well contribute to the exceptionally high KYNA levels which are seen in the fetal brain in several mammalian species [27-31, 44], these findings support the notion that local biochemical events are largely responsible for determining the fate, and therefore the possible function, of KYNA in the fetal brain. Notably, as the blood-brain barrier, which is largely impermeable to KYNA in adulthood [48], is also functional during the prenatal period [55], even relatively large increases in maternal KYNA are unlikely to reach the fetal brain to a significant extent [39].

Considering the possible functional role of KYNA in the prenatal period, and the fact that a variety of stimuli and environmental insults affect KP metabolism in fetal tissues in vivo [44, 56-60], KYNA synthesis from kynurenine was studied in greater detail. In an initial approach, we examined the respective roles of the two bestunderstood KATs, i.e., KAT I and KAT II, by pharmacological means, using AOAA, a non-specific inhibitor of pyridoxal-5-phosphate-dependent enzymes (including all KATs), glutamine (which inhibits KAT I), and the specific KAT II inhibitor BFF-122 as tools [7, 45, 46]. In agreement with reports that KAT II activity is very low in the immature rat brain [28] and plays only a minor role in the adult mouse brain $[7,10]$, these experiments revealed a major role of KAT I in both fetal and maternal 
brain tissue. Assessment of KYNA formation in the placenta, in contrast, did not show a clear difference between KAT I and KAT II, even though only the presence of KAT I has been documented in the placenta previously [52, 61]. As anticipated [7, 41], KYNA neosynthesis in both the maternal and fetal liver was found to be almost exclusively catalyzed by KAT II.

Studies with tissues from KAT II KO mice were performed to complement these preliminary pharmacological studies using a genetic approach. In these experiments, we compared both basal release (i.e., the results of incubation in the absence of kynurenine) with the de novo formation of KYNA from kynurenine using tissues from WT and mutant mice. Generally, these studies confirmed that KYNA synthesis in both maternal and fetal liver is largely catalyzed by KAT II, whereas both basal and stimulated KYNA release in slices from the maternal brain, fetal brain, and placenta is less, or, in the case of basal release from the maternal brain, is not KAT II-dependent.

Together, these findings indicate that the molecular and cellular mechanism(s) that account for KYNA formation during the prenatal period are remarkably complex. Notably, postnatally, the generation of KYNA is influenced by several factors, as KYNA production is stimulated by co-substrates of KAT such as pyruvate or 2-oxoglutarate [62], and inhibited under hypoglycemic conditions and by amino acids that compete with kynurenine as substrates $[63,64]$. However, these regulatory mechanisms have not been examined during gestation. Ongoing studies in our laboratory are also designed to test possible additional contributions of the two KATs that were not examined here (KAT III and KAT IV $[4,7]$ ), and to take into account that KYNA production from kynurenine can also occur non-enzymatically, i.e., by oxidative mechanisms [65]. Moreover, while our study has not indicated significant differences in KYNA formation between tissues collected on GD 14 and GD 18, we are expanding the age range to conduct a comprehensive assessment of KYNA formation during gestation (starting at GD 10) and at several postnatal stages. In these studies, special emphasis will be placed on the developmental pattern of mechanisms that regulate KYNA synthesis and function [62-64] and age-related differences in the metabolite's putative receptor targets $[5,6]$, under both physiological and pathological conditions.

KYNA-producing cells in the prenatal brain deserve particular attention. Although neurons are capable of synthesizing KYNA [66-69], there is consensus that nonneuronal cells, specifically astrocytes, are largely responsible for the de novo formation of KYNA in the mamma- lian brain in adulthood (review [21]). In the developing rodent brain, astrocytes are first detected at GD 16 [70] and continue their maturation in the first postnatal weeks [71]. Perinatally, brain KYNA is therefore probably produced by, and released from, glial progenitor cells and possibly other cell types. Notably, as shown in vitro using human cortical slices obtained at mid-gestation, nanomolar concentrations of KYNA, in turn, affect progenitor cell proliferation, differentiation, and survival [72], and may thus participate actively in cortical circuit formation during brain development [73].

As increased brain KYNA in the fetus and/or in the early postnatal period causes functional and behavioral abnormalities in adulthood [23, 26, 32-35, 37], our findings also have translational implications and relevance for schizophrenia and other brain disorders whose etiology can be traced to events early in life (see Introduction), despite potential differences between mouse and human pregnancy [74]. Thus, clarification of both the enzymatic and nonenzymatic mechanisms that underlie perinatal KYNA formation in different tissues may lead to the conceptualization of fundamentally novel therapeutic interventions. Timely pharmacological treatments designed to mitigate deviant brain KYNA function perinatally may then be targeted to prevent or attenuate the detrimental effects of abnormal KP-related genes [47, 75] and/or early pathogenic insults that affect KP metabolism [44, 52, 56-60].

\section{Statement of Ethics}

All experiments were approved by the Institutional Animal Care and Use Committee (IACUC) of the University of Maryland School of Medicine and followed the "Principles of Laboratory Animal Care" (NIH publication No. 86-23, 1996).

\section{Disclosure Statement}

The authors have no conflicts of interest to declare.

\section{Funding Sources}

This work was supported by USPHS grant MH103222 (Silvio O. Conte Center for Translational Research).

\section{Author Contributions}

F.M.N. and S.B. designed and coordinated the study, prepared the figures and wrote the initial draft of the paper. F.M.N., S.B., and R.S. analyzed and interpreted the data and revised the paper. 


\section{References}

1 Schwarcz R, Bruno JP, Muchowski PJ, Wu HQ. Kynurenines in the mammalian brain: when physiology meets pathology. Nat Rev Neurosci. 2012 Jul;13(7):465-77.

2 Badawy AA. Tryptophan availability for kynurenine pathway metabolism across the life span: control mechanisms and focus on aging, exercise, diet and nutritional supplements. Neuropharmacology. 2017 Jan;112 Pt B:24863.

3 Guidetti P, Hoffman GE, Melendez-Ferro M, Albuquerque EX, Schwarcz R. Astrocytic localization of kynurenine aminotransferase II in the rat brain visualized by immunocytochemistry. Glia. 2007 Jan;55(1):78-92.

4 Han Q, Cai T, Tagle DA, Li J. Structure, expression, and function of kynurenine aminotransferases in human and rodent brains. Cell Mol Life Sci. 2010 Feb;67(3):353-68.

5 Stone TW, Stoy N, Darlington LG. An expanding range of targets for kynurenine metabolites of tryptophan. Trends Pharmacol Sci. 2013 Feb;34(2):136-43.

6 Moroni F, Cozzi A, Sili M, Mannaioni G. Kynurenic acid: a metabolite with multiple actions and multiple targets in brain and periphery. J Neural Transm (Vienna). 2012 Feb; 119(2):133-9.

7 Guidetti P, Amori L, Sapko MT, Okuno E, Schwarcz R. Mitochondrial aspartate aminotransferase: a third kynurenate-producing enzyme in the mammalian brain. J Neurochem. 2007 Jul;102(1):103-11.

8 Turski WA, Gramsbergen JB, Traitler H, Schwarcz R. Rat brain slices produce and liberate kynurenic acid upon exposure to L-kynurenine. J Neurochem. 1989 May;52(5): 1629-36.

9 Swartz KJ, During MJ, Freese A, Beal MF. Cerebral synthesis and release of kynurenic acid: an endogenous antagonist of excitatory amino acid receptors. J Neurosci. 1990 Sep;10(9): 2965-73.

10 Herédi J, Cseh EK, Berkó AM, Veres G, Zádori D, Toldi J, et al. Investigating KYNA production and kynurenergic manipulation on acute mouse brain slice preparations. Brain Res Bull. 2019 Mar;146:185-91.

11 Vécsei L, Szalárdy L, Fülöp F, Toldi J. Kynurenines in the CNS: recent advances and new questions. Nat Rev Drug Discov. 2013 Jan; 12(1):64-82.

12 Heyes MP, Saito K, Crowley JS, Davis LE, Demitrack MA, Der M, et al. Quinolinic acid and kynurenine pathway metabolism in inflammatory and non-inflammatory neurological disease. Brain. 1992 Oct;115(Pt 5): 1249-73.

13 Oxenkrug G. Serotonin-kynurenine hypothesis of depression: historical overview and recent developments. Curr Drug Targets. 2013 May;14(5):514-21.

14 Stone TW. Kynurenines in the CNS: from endogenous obscurity to therapeutic importance. Prog Neurobiol. 2001 Jun;64(2):185-218.
15 Chen Y, Guillemin GJ. Kynurenine pathway metabolites in humans: disease and healthy States. Int J Tryptophan Res. 2009;2:1-19.

16 Schwarcz R, Rassoulpour A, Wu HQ, Medoff D, Tamminga CA, Roberts RC. Increased cortical kynurenate content in schizophrenia. Biol Psychiatry. 2001 Oct;50(7):521-30.

17 Erhardt S, Blennow K, Nordin C, Skogh E, Lindström LH, Engberg G. Kynurenic acid levels are elevated in the cerebrospinal fluid of patients with schizophrenia. Neurosci Lett. 2001 Nov;313(1-2):96-8.

18 Linderholm KR, Skogh E, Olsson SK, Dahl ML, Holtze M, Engberg G, et al. Increased levels of kynurenine and kynurenic acid in the CSF of patients with schizophrenia. Schizophr Bull. 2012 May;38(3):426-32.

19 Sathyasaikumar KV, Stachowski EK, Wonodi I, Roberts RC, Rassoulpour A, McMahon RP, et al. Impaired kynurenine pathway metabolism in the prefrontal cortex of individuals with schizophrenia. Schizophr Bull. 2011 Nov;37(6):1147-56.

20 Erhardt S, Schwieler L, Imbeault S, Engberg G. The kynurenine pathway in schizophrenia and bipolar disorder. Neuropharmacology. 2017 Jan;112 Pt B:297-306.

21 Pocivavsek A, Notarangelo FM, Wu HQ, Bruno JP, Schwarcz R. Astrocytes as Pharmacological Targets in the Treatment of Schizophrenia: Focus on Kynurenic Acid. Modeling the Psychopathological Dimensions of Schizophrenia. San Diego, CA, USA: Elsevier; 2016. pp. 423-43.

22 Akagbosu CO, Evans GC, Gulick D, Suckow RF, Bucci DJ. Exposure to kynurenic acid during adolescence produces memory deficits in adulthood. Schizophr Bull. 2012 Jun;38(4): 769-78.

23 Asp L, Holtze M, Powell SB, Karlsson H, Erhardt S. Neonatal infection with neurotropic influenza A virus induces the kynurenine pathway in early life and disrupts sensorimotor gating in adult Tap1-/- mice. Int J Neuropsychopharmacol. 2010 May;13(4):47585.

24 Erhardt S, Pocivavsek A, Repici M, Liu XC, Imbeault S, Maddison DC, et al. Adaptive and Behavioral Changes in Kynurenine 3-Monooxygenase Knockout Mice: Relevance to Psychotic Disorders. Biol Psychiatry. 2017 Nov; 82(10):756-65.

25 DeAngeli NE, Todd TP, Chang SE, Yeh HH, Yeh PW, Bucci DJ. Exposure to Kynurenic Acid during Adolescence Increases SignTracking and Impairs Long-Term Potentiation in Adulthood. Front Behav Neurosci. 2015 Jan;8:451.

26 Pocivavsek A, Thomas MA, Elmer GI, Bruno JP, Schwarcz R. Continuous kynurenine administration during the prenatal period, but not during adolescence, causes learning and memory deficits in adult rats. Psychopharmacology (Berl). 2014 Jul;231(14):2799809.
27 Beal MF, Swartz KJ, Isacson O. Developmental changes in brain kynurenic acid concentrations. Brain Res Dev Brain Res. 1992 Jul;68(1):136-9.

28 Ceresoli-Borroni G, Schwarcz R. Perinatal kynurenine pathway metabolism in the normal and asphyctic rat brain. Amino Acids. 2000;19(1):311-23

29 Cannazza G, Chiarugi A, Parenti C, Zanoli P, Baraldi M. Changes in kynurenic, anthranilic, and quinolinic acid concentrations in rat brain tissue during development. Neurochem Res. 2001 May;26(5):511-4.

30 Notarangelo FM, Pocivavsek A. Elevated kynurenine pathway metabolism during neurodevelopment: implications for brain and behavior. Neuropharmacology. 2017 Jan;112 Pt B:275-85.

31 Walker DW, Curtis B, Lacey B, Nitsos I. Kynurenic acid in brain and cerebrospinal fluid of fetal, newborn, and adult sheep and effects of placental embolization. Pediatr Res. 1999 Jun; 45(6):820-6.

32 Pershing ML, Bortz DM, Pocivavsek A, Fredericks PJ, Jørgensen CV, Vunck SA, et al. Elevated levels of kynurenic acid during gestation produce neurochemical, morphological, and cognitive deficits in adulthood: implications for schizophrenia. Neuropharmacology. 2015 Mar;90:33-41.

33 Forrest CM, Khalil OS, Pisar M, McNair K, Kornisiuk E, Snitcofsky M, et al. Changes in synaptic transmission and protein expression in the brains of adult offspring after prenatal inhibition of the kynurenine pathway. Neuroscience. 2013 Dec;254:241-59.

34 Khalil OS, Pisar M, Forrest CM, Vincenten MC, Darlington LG, Stone TW. Prenatal inhibition of the kynurenine pathway leads to structural changes in the hippocampus of adult rat offspring. Eur J Neurosci. 2014 May; 39(10):1558-71.

35 Pisar M, Forrest CM, Khalil OS, McNair K, Vincenten MC, Qasem S, et al. Modified neocortical and cerebellar protein expression and morphology in adult rats following prenatal inhibition of the kynurenine pathway. Brain Res. 2014 Aug;1576:1-17.

36 Pershing ML, Phenis D, Valentini V, Pocivavsek A, Lindquist $\mathrm{DH}, \mathrm{Schwarcz} \mathrm{R}$, et al. Prenatal kynurenine exposure in rats: age-dependent changes in NMDA receptor expression and conditioned fear responding. Psychopharmacology (Berl). 2016 Oct;233(2122):3725-35.

37 Alexander KS, Pocivavsek A, Wu HQ, Pershing ML, Schwarcz R, Bruno JP. Early developmental elevations of brain kynurenic acid impair cognitive flexibility in adults: reversal with galantamine. Neuroscience. 2013 May; 238:19-28.

38 Nilsen RM, Bjørke-Monsen AL, Midttun O, Nygård O, Pedersen ER, Ulvik A, et al. Maternal tryptophan and kynurenine pathway metabolites and risk of preeclampsia. Obstet Gynecol. 2012 Jun;119(6):1243-50. 
39 Goeden N, Notarangelo FM, Pocivavsek A, Beggiato S, Bonnin A, Schwarcz R. Prenatal dynamics of kynurenine pathway metabolism in mice: focus on kynurenic acid. Dev Neurosci. 2017;39(6):519-28.

40 Nicholls T, Nitsos I, Walker DW. Tryptophan metabolism in pregnant sheep: increased fetal kynurenine production in response to maternal tryptophan loading. Am J Obstet Gynecol. 1999 Dec;181(6):1452-60.

41 Yu P, Di Prospero NA, Sapko MT, Cai T, Chen A, Melendez-Ferro M, et al. Biochemical and phenotypic abnormalities in kynurenine aminotransferase II-deficient mice. Mol Cell Biol. 2004 Aug;24(16):6919-30.

42 Boisset JC, Andrieu-Soler C, van Cappellen WA, Clapes T, Robin C. Ex vivo time-lapse confocal imaging of the mouse embryo aorta. Nat Protoc. 2011 Oct;6(11):1792-805.

43 Lowry OH, Rosebrough NJ, Farr AL, Randall RJ. Protein measurement with the Folin phenol reagent. J Biol Chem. 1951 Nov;193(1): 265-75.

44 Notarangelo FM, Schwarcz R. Restraint Stress during Pregnancy Rapidly Raises Kynurenic Acid Levels in Mouse Placenta and Fetal Brain. Dev Neurosci. 2016;38(6):458-68.

45 Amori L, Guidetti P, Pellicciari R, Kajii Y, Schwarcz R. On the relationship between the two branches of the kynurenine pathway in the rat brain in vivo. J Neurochem. 2009 Apr; 109(2):316-25.

46 Speciale C, Wu HQ, Gramsbergen JB, Turski WA, Ungerstedt U, Schwarcz R. Determination of extracellular kynurenic acid in the striatum of unanesthetized rats: effect of aminooxyacetic acid. Neurosci Lett. 1990 Aug; 116(1-2):198-203.

47 Beggiato S, Notarangelo FM, Sathyasaikumar KV, Giorgini F, Schwarcz R. Maternal genotype determines kynurenic acid levels in the fetal brain: implications for the pathophysiology of schizophrenia. J Psychopharmacol. 2018 Nov;32(11):1223-32.

48 Fukui S, Schwarcz R, Rapoport SI, Takada Y, Smith QR. Blood-brain barrier transport of kynurenines: implications for brain synthesis and metabolism. J Neurochem. 1991 Jun; 56(6):2007-17.

49 Chrostowski MK, McGonnigal BG, Stabila JP, Padbury JF. LAT-1 expression in pre- and post-implantation embryos and placenta. Placenta. 2009 Mar;30(3):270-6.

50 Badawy AA. Tryptophan metabolism, disposition and utilization in pregnancy. Biosci Rep. 2015 Sep;35(5):35.

51 Ligam P, Manuelpillai U, Wallace EM, Walker D. Localisation of indoleamine 2,3-dioxy- genase and kynurenine hydroxylase in the human placenta and decidua: implications for role of the kynurenine pathway in pregnancy. Placenta. 2005 Jul;26(6):498-504.

52 Manuelpillai U, Ligam P, Smythe G, Wallace EM, Hirst J, Walker DW. Identification of kynurenine pathway enzyme mRNAs and metabolites in human placenta: up-regulation by inflammatory stimuli and with clinical infection. Am J Obstet Gynecol. 2005 Jan; 192(1):280-8

53 Alberati-Giani D, Ricciardi-Castagnoli P, Köhler C, Cesura AM. Regulation of the kynurenine metabolic pathway by interferongamma in murine cloned macrophages and microglial cells. J Neurochem. 1996 Mar; 66(3):996-1004.

54 Murthi P, Wallace EM, Walker DW. Altered placental tryptophan metabolic pathway in human fetal growth restriction. Placenta. 2017 Apr;52:62-70.

55 Goasdoué K, Miller SM, Colditz PB, Björkman ST. Review: the blood-brain barrier; protecting the developing fetal brain. Placenta. 2017 Jun;54:111-6.

56 Notarangelo FM, Schwarcz R. Prenatal LPS exposure preferentially increases kynurenine pathway metabolism in the fetal brain. Soc Neurosci. 2015;40:74.

57 Nicholls T, Nitsos I, Smythe G, Walker DW. Kynurenine production and catabolism in fetal sheep with embolized or nonembolized placentas. Am J Obstet Gynecol. 2001 Oct; 185(4):988-95.

58 Sano M, Ferchaud-Roucher V, Kaeffer B, Poupeau G, Castellano B, Darmaun D. Maternal and fetal tryptophan metabolism in gestating rats: effects of intrauterine growth restriction. Amino Acids. 2016 Jan;48(1):28190.

59 Baran H, Kepplinger B, Herrera-Marschitz M, Stolze K, Lubec G, Nohl H. Increased kynurenic acid in the brain after neonatal asphyxia. Life Sci. 2001 Aug;69(11):1249-56.

60 Ceresoli-Borroni G, Schwarcz R. Neonatal asphyxia in rats: acute effects on cerebral kynurenine metabolism. Pediatr Res. 2001 Aug; 50(2):231-5.

61 Milart P, Urbanska EM, Turski WA, Paszkowski T, Sikorski R. Kynurenine aminotransferase I activity in human placenta. Placenta. 2001 Feb-Mar;22(2-3):259-61.

62 Hodgkins PS, Wu HQ, Zielke HR, Schwarcz R. 2-Oxoacids regulate kynurenic acid production in the rat brain: studies in vitro and in vivo. J Neurochem. 1999 Feb;72(2):643-51.

63 Gramsbergen JB, Hodgkins PS, Rassoulpour A, Turski WA, Guidetti P, Schwarcz R. Brain- specific modulation of kynurenic acid synthesis in the rat. J Neurochem. 1997 Jul;69(1): 290-8.

64 Schwarcz R, Poeggeler B, Rassoulpour A, Ceresoli-Borroni G, Hodgkins PS. Regulation of kynurenic acid levels in the developing rat brain. Amino Acids. 1998;14(1-3):243-9.

65 Blanco Ayala T, Lugo Huitrón R, Carmona Aparicio L, Ramírez Ortega D, González Esquivel D, Pedraza Chaverrí J, et al. Alternative kynurenic acid synthesis routes studied in the rat cerebellum. Front Cell Neurosci. 2015 May;9:178.

66 Du F, Schmidt W, Okuno E, Kido R, Köhler C, Schwarcz R. Localization of kynurenine aminotransferase immunoreactivity in the rat hippocampus. J Comp Neurol. 1992 Jul; 321(3):477-87.

67 Guillemin GJ, Cullen KM, Lim CK, Smythe GA, Garner B, Kapoor V, et al. Characterization of the kynurenine pathway in human neurons. J Neurosci. 2007 Nov;27(47):12884-92.

68 Herédi J, Berkó AM, Jankovics F, Iwamori T, Iwamori N, Ono E, et al. Astrocytic and neuronal localization of kynurenine aminotransferase- 2 in the adult mouse brain. Brain Struct Funct. 2017 May;222(4):1663-72.

69 Roberts RC, Du F, McCarthy KE, Okuno E, Schwarcz R. Immunocytochemical localization of kynurenine aminotransferase in the rat striatum: a light and electron microscopic study. J Comp Neurol. 1992 Dec;326(1):8290.

70 Bayraktar OA, Fuentealba LC, Alvarez-Buylla A, Rowitch DH. Astrocyte development and heterogeneity. Cold Spring Harb Perspect Biol. 2014 Nov;7(1):a020362.

71 Semple BD, Blomgren K, Gimlin K, Ferriero DM, Noble-Haeusslein LJ. Brain development in rodents and humans: identifying benchmarks of maturation and vulnerability to injury across species. Prog Neurobiol. 2013 Jul-Aug;106-107:1-16.

72 Bagasrawala I, Zecevic N, Radonjić NV. NMethyl D-Aspartate Receptor Antagonist Kynurenic Acid Affects Human Cortical Development. Front Neurosci. 2016 Sep;10:435.

73 Allen NJ, Lyons DA. Glia as architects of central nervous system formation and function. Science. 2018 Oct;362(6411):181-5.

74 Clark DA. The use and misuse of animal ana$\log$ models of human pregnancy disorders. J Reprod Immunol. 2014 Jun;103:1-8.

75 Boros FA, Bohár Z, Vécsei L. Genetic alterations affecting the genes encoding the enzymes of the kynurenine pathway and their association with human diseases. Mutat Res. 2018 Apr - Jun;776:32-45. 